\title{
Estrategia de gestión de servicios de voz y datos sobre Internet
}

\author{
Fecha de recepción: 04/10/2019 • Fecha de aceptación: 02/12/2019 • Fecha de publicación:10/02/2020
}

David Iván Collaguazo

Corporación Nacional de Telecomunicaciones

dacollaguazo@hotmail.com

https://orcid.org/0000-0003-3483-0099

\section{RESUMEN}

La presente investigación presenta la implementación de una Estrategia de Gestión de Servicios de Voz y Datos sobre Internet, para lo cual se utilizó un diseño de campo, el estudio se realizó in situ, con base a estos resultados se logró determinar la condición de mayor incidencia negativa en la empresa es que esta no cuenta una estrategia de gestión para dar respuesta inmediata y eficaz al daño que pueden causar en las transmisiones cuando son afectadas por agentes externos e internos fortuitos. La investigación se realizó dentro de la Corporación Nacional de Telecomunicaciones, siendo esta la locación donde se alojan las transmisiones de Quito, Guayaquil y Cuenca. La metodología de trabajo se basó en ITIL, siendo la más apropiada en la fase de seguimiento, gestión e implementación según las normas establecidas por la corporación. En función de la metodología utilizada conllevo al desarrollo de una estrategia de modelo inclusivo, alineado con la normativa de la corporación

\section{PALABRAS CLAVE: estrategias de gestión, servicios de voz y datos, internet}

\section{ABSTRACT}

The present investigation presents the implementation of a Strategy of Management of Services of Voice and Data on Internet, for which a field design was used, the study was realized in situ, based 
on these results it was possible to determine the condition of major negative incidence in the company is that this one does not count with a strategy of management to give immediate and effective response to the damage that can cause in the transmissions when they are affected by external and internal fortuitous agents. The investigation was carried out within the National Telecommunications Corporation, which is the location where the transmissions from Quito, Guayaquil and Cuenca are housed. The work methodology was based on ITIL, being the most appropriate in the phase of monitoring, management and implementation according to the rules established by the corporation. Depending on the methodology used, it led to the development of an inclusive model strategy, aligned with the corporation's regulations

KEYWORDS: management strategies, voice and data services, internet 


\section{INTRODUCCIÓN}

Las Estrategias de Gestión de Servicios, en el ámbito de las telecomunicaciones, es un recurso dinámico, que se enfoca principalmente en asignar tareas directas a ejecutar acciones de acuerdo a la responsabilidad otorgada en la cadena de mando, a fin de obtener la mejoras en los servicios y de esta forma satisfacer a los clientes, por lo que el objetivo de esta investigación es implementar una estrategia de gestión de servicios de voz y datos sobre el internet.

Uno de los enfoques de las Estrategias de Gestión de Servicios es la adaptación, es decir, ir creciendo en capas, con la finalidad de poder configurarse a su entorno. De este modo, con las capacidades antes dispuestas, se puede enfocar cada una de las transmisiones afectadas y estudiar cada uno de sus comportamientos.

Por tanto, una Estrategia de Gestión de Servicios, debe adaptarse a cada una de las características de transmisiones en base con el nivel de funcionamiento de las mismas, esto con la finalidad de interactuar entre la estrategia de gestión, el sistema y el autor. Los pasos establecidos a seguir para la revisión e implementación de los procesos que deben tener cada una de las transmisiones en base al nivel de su funcionamiento, esto con la finalidad de interactuar entre la estrategia de gestión, el proveedor y sus directrices.

De acuerdo a lo planteado se puede mencionar que el nacimiento de la propuesta; estrategias de gestión de servicios de Voz y Datos sobre Internet, es un recurso innovador, cuyo propósito es conformar una alianza entre el sistema actual y las posibles afectaciones en el transcurso de tiempo, donde las peticiones actuales y antiguas se integran, dando así un nuevo enfoque a los procesos actuales.

Por otra parte, la Corporación Nacional de Telecomunicaciones como empresa gubernamental que se dedica a proveer diferentes servicios al país, con el fin de estar a la vanguardia de las comunicaciones, dentro de su cartera de servicios cuentan con la tecnología Red Óptica Pasiva con Capacidad de Gigabit GPON, sin duda actualmente es la que más se destaca en el mercado con su internet residencial disminuyendo los costos de implementación y con velocidades superiores a los 5 megas, sin embargo, por tener un alto consumo de tráfico en dicha tecnología, la red se hace lenta, se pierde la conexión y la respuesta para restablecer el servicio es lenta, por lo que es necesario disponer de transmisiones que soporten ese tráfico.

En base a lo mencionado, se realizará el estudio de una estrategia de gestión en los servicios de voz y datos sobre el internet, el cual permita proporcionar un proceso adecuado y automatizado en base a los lineamientos de la corporación para solventar el problema de afectación en el tráfico que circula en ese momento por las transmisiones, y permite fusionar la parte tecnológica con los eventos, destacando el propósito de fortalecer el área del monitoreo de red e impulsar la revisión de cada una de sus transmisiones y así evitar que poblaciones enteras se queden sin servicio, mejorando así el servicio a los usuarios y colocándose a la par de sus competidores. Cabe destacar que el apoyo tecnológico fomenta el soporte continuo mediante la construcción de nuevos canales de comunicación que se suman con los existentes, mejorando considerablemente la calidad de servicio y satisfacción de los clientes. 
Después de argumentar los problemas que actualmente se tienen, se plantea la interrogante con respecto a si una estrategia de gestión de servicios ¿podría mejorar los procesos preestablecidos en el área de O\&M cumpliendo así los SLA con los usuarios corporativos y masivos finales?

En función a lo anterior, la presente investigación, se justifica pues la misma pretende crear una manera de gestionar y organizar los procesos de revisión para las transmisiones al momento de tener problemas y saber cómo solucionarlos, lo cual traerá grandes beneficios a la empresa desde el punto de vista de reducción de tiempo y costos para restablecimiento de la conexión, coadyuvando de esta manera a la satisfacción del cliente, al poseer un servicio eficaz y eficiente. Cabe destacar, que la implementación de la estrategia de gestión se hará con el fin de tener pasos concretos y normas para que los clientes no pierdan la información que se encuentra circulando por estas, cabe mencionar que también la presente investigación se justifica pues servirá como base para otras investigaciones similares en las cuales se pretendan mejorar los tiempos de respuestas ante fallas que se presenten en sus transmisiones dentro de su infraestructura todo esto con el fin de proporcionar a las empresas la factibilidad de tener acciones de corrección de pronta reacción y así o afectar al usuario final.

Para solucionar el problema planteado, se hicieron diferentes reuniones con el departamento técnico, con la finalidad de determinar los principales problemas que afectan el tráfico en la RED de CNT, llegando a varios acuerdos establecidos entre el investigador y las personas que se encargan de administrar los contratos en donde basándose en las normas ya establecidas en la CNT se debe realizar la implementación de la estrategia enmarcada en las normas de calidad de servicio que se encuentran aplicadas dentro de la CNT, tomando en cuenta que al momento de llevar a cabo la presente factibilidad se tuvo la necesidad de los Analistas de Gestión que laboran dentro del área de monitoreo de red, realizando el seguimiento exhaustivo de los resultados que proporcione la estrategia.

El estudio pretende crear una estrategia de gestión que permita mejorar la transmisión de voz y datos sobre Internet exceptuando la telefonía móvil, en la ciudad de Quito, estará enmarcado dentro de la innovación de las estrategias de gestión, en el área de la tecnología de información y comunicación, concretamente, en los sistemas de transmisión tanto de voz como datos mediante la Internet, con la finalidad de mejorar el tiempo de respuesta al momento de ocurrir una falla del servicio, cuando las mismas impliquen corte de fibra, saturación del canal, intermitencia en el servicio, tomando como base los aportes de Prieto y otros (2016), González (2014), Bastidas (2016), Lorenzo (2012), Rodríguez (2017) entre otros.

En relación a lo anterior, se utilizó el método de cascada el cual permitió realizar los siguientes pasos: requisitos, diseño, implementación, verificación y mantenimiento. También se realizó la consulta a expertos que se encuentran dentro de la CNT en donde se tuvo la necesidad de la elaboración de una estrategia de gestión elaborado con la metodología de cascada de acuerdo a (Royce, 1970), para esto se adaptaron cinco fases como son: análisis, diseño, desarrollo, producción y evaluación, a continuación, se detallan cada una de las fases 
Toda investigación requiere de la exploración de referentes teóricos, que puedan servir de aporte con el que se pretende desarrollar, razón por la cual se hizo una revisión exhaustiva, donde se encontraron conceptos relacionados con las variables en estudio: Estrategia de Gestión y Servicios de voz y datos sobre Internet.

Con respecto a la variable estrategia de gestión se afirma que una estrategia es el conjunto de decisiones fijadas en un determinado contexto o plano que proceden del proceso organizacional y que integra misión, objetivos y secuencia de acciones administrativas en un todo independiente. Además, en su artículo Lorenzo (2012) menciona, una estrategia es la forma en que la empresa o institución en relación con todo su entorno se debe desplegar todos los recursos disponibles y esfuerzos para alcanzar sus metas u objetivos.

\section{Transmisión de voz sobre datos}

Joskowicz (2013) menciona que para lograr transmitir la voz sobre las redes de datos es necesario armar paquetes, un canal de voz tiene un flujo de bits dependiendo del codec utilizado. Sin embargo, se puede armar un paquete por cada flujo de voz, pero no es lo aconsejable debido a que esto generaría una sobrecarga (overhead) cabe mencionar algo muy importante que cada paquete requiere cabezas, en cambio para formar paquetes con una mínima sobrecarga pueden introducirse retrasos no muy aceptables.

\section{Datos}

Los datos se representan mediante manera simbólica y este es un valor que puede recibir un computador por diferentes medios de transmisión, los datos solos no pueden proporcionar información cuando estos forman una red de datos es ahí donde se considera una fuente de información.

\section{Redes de datos}

Las redes de datos tienen como objetivo:

- Compartir recursos, equipos, información y programas que se encuentran localmente y ubicados en distintos lugares.

- Brindar confiabilidad a la información disponiendo de alternativas de almacenamiento.

- Obtener una relación aceptable del costo y beneficio.

- Transmitir información entre usuarios ubicados en distintos lugares geográficos de la manera más rápida y eficiente posible. 


\section{Estrategia de gestión de servicios de voz y datos sobre internet}

Una estrategia de gestión de servicios de voz y datos sobre internet se puede definir como la forma de realizar el cambio de un proceso de los servicios orientados a la voz y datos dentro de una organización con la ayuda de sus metas u objetivos trazados, analizando la mejora de la capacidad del ancho de banda y controlando la calidad de servicio dentro de los parámetros que sean manejables para la organización sin afectar los tiempos de respuesta que se tengan dentro de las áreas involucradas, además de tomar en cuenta los dispositivos que la empresa dispone en el momento de realizar la investigación para en lo posterior sugerir mantener o actualizar.

\section{Operación de servicio}

Representa un proceso de ITIL para asegurar que los servicios se ofrezcan efectiva y eficientemente, esto incluye cumplir con los requerimientos de los usuarios, resolver los fallos de los servicios, arreglar problemas y llevar a cabo operaciones rutinarias, La rutina operación de servicio abarca los procesos siguientes: gestión de eventos, gestión de incidentes, cumplimiento de la solicitud, gestión de acceso, gestión de problemas, gestión de operaciones y gestión de instalaciones.

\section{Situación actual de la gestión de servicios de voz y datos sobre internet}

Actualmente los servicios de voz y datos que proporciona la corporación nacional de telecomunicaciones se encuentran vulnerados a través de sus terminales debido al encontrarse con malware que son activados por los hackers y esto provoca que la transmisión que lleva a los DNS (DomainNameSystem) se encuentre saturada provocando lentitud en el servicio de datos e internet en la ciudad de Quito, sin embargo, se activa manualmente la política de levantamiento de la transmisión que se encuentra dirigida hacia los servidores que se encuentran en Guayaquil.

Es decir, las transmisiones que se encuentran dentro del distrito metropolitano de Quito disponen de un enlace de respaldo, lo cual no sucede con los barrios que se encuentran a los alrededores de la ciudad y llevan el tráfico de voz y datos hacia las diferentes ciudades con el fin de que se encuentren conectadas y no se presenten percances cuando sufran algún corte de fibra y no se pueda respaldar el tráfico. Por tanto se quiere proponer una estrategia de gestión de servicios para las transmisiones que se encuentren saturadas y se pueda revisar e informar al área encargada para que esta proceda con el balanceo del tráfico respectivo en casa de que alguna de estas transmisiones se encuentre ocupando sobre el $85 \%$ de consumo y en caso de existir algún corte de fibra enviar a las cuadrillas a solventar el problema mientras el tráfico e encuentre balanceado, de tal manera, se necesita realizar el estudio de las transmisiones que se encuentran sin respaldo y proponer estrategias de ayuda y soporte.

La presente investigación es, según la tipificación realizada por Hernández, Fernández y Baptista (2006) descriptiva, ya que se refiere a la descripción, registro, análisis e interpretación de la naturaleza actual y los procesos de los fenómenos estudiados, relacionados con la necesidad 
del diseño de una Estrategia de Gestión de Servicios de Voz y Datos sobre Internet. En cuanto al diseño de la investigación González y otros (2016, p. 63), lo definen como "la proyección de la investigación la toma de decisiones sobre el qué y el cómo se va a realizar".

De igual forma; Arias (2000), expone que esta fase se refiere a donde se puede ubicar la investigación de acuerdo al método o al tipo de metodología empleada, aplicando las técnicas y procedimientos que sean necesarios para llegar a la culminación de la investigación planteada.

De tal manera, Hernández y otros (2006, p.208) indican que la "investigación transaccional o transversa recolectan datos en un solo momento, en un tiempo único", considerando lo expuesto la presente investigación se encuentra orientada en sus objetivos, todo esto con la finalidad de que se presenten una o más variables en el transcurso del tiempo para continuar con el análisis en un momento determinado; es decir, tiene como objetivo verificar la incidencia y los valores de las variables para proporcionar la descripción de la información.

La población de una investigación, según Tamayo (2007, p. 176) en su libro menciona, "se define como población por constituir la totalidad del fenómeno adscrito a un estudio o investigación", es decir todo el fenómeno que se encuentre dentro de una investigación y que pueda proporcionar información relevante se la considera población, de la misma manera.

En este sentido, se tomó una muestra no probabilística de seis (6) sujetos de estudio, supervisores y analistas especialistas que laboran en el área de monitoreo de red por conveniencia del investigador, estas fueron seleccionadas de forma intencional, por ser una población de fácil acceso, por tener la característica común de ser integrantes activos de la gerencia nacional técnica, por estar expuestos en su trabajo a la problemática desde el comienzo hasta el fin del proyecto de investigación. Por tal motivo, la muestra no probabilística intencional seleccionada se puede observar en la Tabla 1 siguiente:

Tabla 1

Muestra no probabilística intencional

\begin{tabular}{lll}
\hline Descripción & Supervisor & Analistas \\
\hline Administrar el TR69 & 1 & 1 \\
\hline Comunicar disponibilidad y desempeño de la red & 1 & 1 \\
\hline Administrar usuarios y accesos & 1 & 1 \\
\hline Subtotal & 3 & 3 \\
\hline Total de sujetos & 6 & \\
\hline
\end{tabular}

Después de mencionar y definir los procesos metodológicos de investigación, se debe seleccionar las técnicas e instrumentos de recolección de datos que se van a utilizar, Sabino $(2007$, p. 108) "cualquier recurso de los que se vale el investigador para acercarse a los fenómenos y extraer información", de igual manera para Arias (2012, p. 111), las técnicas e instrumentos de recolección de datos son las distintas formas de obtener información y los instrumentos son los medios 
materiales, en base a lo mencionado se puede concluir que la técnica y el instrumento de la encuesta es el modo y el medio respectivamente con el que un investigador puede acceder a una recopilación de datos.

Así también, de acuerdo a Murillo (2006, p.12) las escalas "ofrece una afirmación al sujeto y pide que se califique del 0 al 4 según su grado de conformidad, esta afirmación puede reflejar la actitud positiva o negativa hacia algo", esta herramienta es la encargada de medir actitudes individuales en contextos sociales específicos.

Por lo tanto, a cada uno de estos ítems del instrumento elaborado se le coloco una ponderación, 4, $3,2,1$, de acuerdo a la alternativa de respuesta seleccionada las alternativas de respuesta fueron, De Acuerdo, Algo de Acuerdo, Desacuerdo y Completamente Desacuerdo, respectivamente, por lo que cada persona le dio un valor con respecto a su experiencia o conocimiento de la variable en estudio, obteniendo la puntuación total a través de la sumatoria de todas las respuestas. Como consecuencia, para este proyecto fue necesario tomar de referencia el siguiente baremo de la Tabla 3 para poder ordenar y tabular la información:

Tabla 3. Escala de valores

\begin{tabular}{ll}
\hline Valores & Escala de respuestas \\
\hline $1,00 \leq X<1,99$ & Inadecuado \\
\hline $2,00 \leq X<2,99$ & $\begin{array}{l}\text { Moderadamente } \\
\text { Inadecuado }\end{array}$ \\
\hline $3,00 \leq X<3,99$ & Moderadamente \\
& Adecuado \\
\hline$X=4$ & Adecuado \\
\hline
\end{tabular}

\section{RESULTADOS Y CONCLUSIONES}

Para análisis de resultados fue necesario desarrollar una media aritmética individual y media aritmética de la totalidad del resultado además del promedio de los indicadores, esto se realizó separando en dos grupos de seis preguntas donde el primer grupo se evalúa el proceso de revisión que actualmente posee la corporación, el segundo grupo evalúa la gestión que realiza con el proceso anterior y el último grupo de tres preguntas del cuestionario es el cual determina el proceso de implementación de la estrategia.

En base a la descripción actual de la gestión de servicios de voz y datos que se encuentran dentro de la CNT, se pudo constatar que no se encontró una implementación de estrategias sobre las transmisiones para la ciudad de Quito; por lo tanto, fue una necesidad por parte de la corporación el saber cómo se encuentran organizados y estructurados los servicios de voz y datos sobre el internet.

Dentro de los elementos que se tomaron como referencia para poder realizar la investigación de la 
estrategia de gestión de servicios de voz y datos sobre internet se encuentra el macro proceso de ITIL llamado Operación de Servicios con el cual se pudieron profundizar en todos los sub-procesos que este contiene, teniendo así un soporte de una metodología fuerte para el desarrollo de procesos en cuanto se refiere a modelos de trabajo aplicados dentro de la corporación, cada uno de estos sub procesos contribuyeron de una manera asociativa aumentando la productividad en el área de monitoreo de red teniendo en cuenta los resultados de las transmisiones que ya no se tenían saturaciones y los servicios mejoraron notablemente.

En base a lo anterior, se evidencia que cuando los servicio se encuentran con intermitencia o varias transmisiones cae por algún factor externo no cuentan con el debido respaldo y los tiempos exactos de respuesta. También, se logró establecer la estrategia de gestión de servicios de voz y datos sobre internet esté se encuentra el macro proceso de ITIL llamado Operación de Servicios con el cual se pudieron profundizar en todos los sub-procesos que este contiene, teniendo así un soporte de una metodología fuerte para el desarrollo de procesos en cuanto se refiere a modelos de trabajo aplicados dentro de la corporación, cada uno de estos sub procesos contribuyeron de una manera asociativa aumentando la productividad en el área de monitoreo de red teniendo en cuenta los resultados de las transmisiones que ya no se tenían saturaciones y los servicios mejoraron notablemente.

Por otra parte, se detectaron inconvenientes que no solo se generaban por la no pronta conmutación de las transmisiones las cuales se detallan a continuación: falta de seguridad en los puertos de los módems, tarjetas de los equipos de transmisión obsoletas, no se dispone de repuestos para las radios, intermitencia en el servicio de internet para clientes residenciales, centrales con poca climatización para equipos robustos. Con las referencias anteriores se hizo énfasis en el diseño de la estrategia para el restablecimiento de las transmisiones sin prolongar el daño que estas produzcan, en base a lo antes mencionado se logró hacer el diseño.

Sin embargo, por tratarse de una entidad gubernamental la creación de la estrategia depende de muchos factores teniendo presente que se debe seguir el orden jerárquico que dispone la corporación para se pueda realizar un análisis del documento realizado y este pase al departamento de implementación, en donde ahí se pueda dar el paso siguiente que es la aceptación del documento y posterior la mejora del proceso. Sin embargo, hasta el momento se pudo crear y entregar la estrategia en donde consta el recibido por la persona responsable del área de monitoreo de red.

Finalmente la evaluación de la estrategia de gestión de servicios de voz y datos sobre internet se pudo realizar exitosamente con las personas experimentadas considerando que son las mismas que proporcionaron los datos para poder realizar el diseño de la estrategia, con esto se pudo ratificar que la estrategia puede ser implementada ya que fueron tomados todas las falencias que hasta el momento del desarrollo de la investigación se encontraban, es por eso que los procesos redactados en dicha estrategia abarcan los problemas que fueron encontrados en base al problema que se tiene con las transmisiones, con lo cual se considera que la estrategia tuvo una evaluación exitosa. 


\section{REFERENCIAS BIBLIOGRÁFICAS}

Alvear, B., \& Rafael, E. (2016). Análisis y evaluación de la gestión de proyectos de inversión del Sistema Nacional de Transmisión eléctrica del Ecuador realizados entre los años 2010 a 2014 y propuesta de mejoramiento de la dirección de proyectos (Master'sthesis, Quito, 2016.).

Arias, F. (2006). El Proyecto de Investigación. Venezuela: Episteme.

Bustos, J. F., Sepúlveda, A. L., \& Triviño, L. K. (2014). Estrategias del sector de telefonía pública básica conmutada frente al actual desplazamiento de formas tradicionales de comunicación en Colombia (Strategies of theCommuted Basic PublicPhone in theFace of theCurrentDisplacement of TraditionalComunicationFor$\mathrm{ms}$ in Colombia).

Carballar, J. A. (2008). VolP la lelfonía de internet. Madrid: Thomson Editores Spain.

Dominguez, P. R. (09 de 2007). Introducción a la Gestión Empresarial. Recuperado el 23 de 05 de 2018, de http://www.adizesca.com/site/assets/g- introduccion_a_la_gestion_empresarial-pr.pdf

Eveliux.com. (07 de September de 2015). Eveliux.com. Recuperado el 23 de Junio de 2018, de http://www.eveliux.com/mx/ancho-de-banda- definicion.html

González Huerta, M. G. (2014). Imagen corporativa en instituciones públicas de la ciudad de Quito; caso de estudio: Corporación Nacional de Telecomunicaciones (CNT)-Análisis de estrategias vinculadas al cliente interno (Master'sthesis, Universidad Andina Simón Bolívar, Sede Ecuador).

Goode, B. (September de 2002). Voice Over Internet Protocol (VoIP). California, EEUU: Paper.

Hernández, R., Fernández, C., \& Baptista, P. (2006). Metodología de la Investigación. México: McGRAWHILLIINTERAMERICMA EDITORES, SA DE C.V.

Hurtado, J. (2010). Guía para la compresnsión holística de la Ciencia. Caracas: Fundación Sypal.

Joskowicz, D. J. (Agosto de 2013). Voz y Video en Redes IP. Montevideo, Uruguay: Universidad de la República.

Joskowicz, J. (Agosto de 2008). Redes de Datos. Recuperado el 23 de 05 de 2018, de https://www.researchgate.net/publication/26690771 4_REDES_DE_DATOS

Joskowicz, D. J. (12 de Agosto de 2013). Voz, Video y Telonía sobre IP. Montevideo, Uruguay: Universidad República de Uruguay.

Lorenzo, A. F. (Enero de 2012). Conceptos de Estrategia Empresarial. Madrid, España: Escuela de Organización Industrial. 
Prieto, I., Paco, J., Martinez, A., \& Solórzano, L. F. (2016). Análisis de Las Estrategias Públicas Para el Desarrollo del Internet Móvil en Las Comunidades 